\title{
openheart Sibling history is associated with heart failure after a first myocardial infarction
}

\author{
Charlotte Glinge (1) , , ${ }^{1,2}$ Louise Oestergaard, ${ }^{3,4}$ Reza Jabbari, ${ }^{1}$ Sara Rossetti, ${ }^{1}$ \\ Regitze Skals, ${ }^{4}$ Lars Køber, ${ }^{1}$ Thomas Engstrøm, ${ }^{1,5}$ Connie R Bezzina, ${ }^{2}$ \\ Christian Torp-Pedersen, ${ }^{4}$ Gunnar Gislason, ${ }^{3,6,7}$ Jacob Tfelt-Hansen ${ }^{1,8}$
}

\begin{abstract}
- Additional material is published online only. To view, please visit the journal online (http://dx.doi.org/10.1136/ openhrt-2019-001143).
\end{abstract}

To cite: Glinge C, Oestergaard L, Jabbari $\mathrm{R}$, et al. Sibling history is associated with heart failure after a first myocardial infarction. Open Heart 2020;7:e001143. doi:10.1136/ openhrt-2019-001143

CT-P, GG and JT-H contributed equally.

CT-P, GG and JT-H are joint senior authors.

Received 26 July 2019 Revised 20 November 2019 Accepted 26 November 2019

Check for updates

(C) Author(s) (or their employer(s)) 2020. Re-use permitted under CC BY-NC. No commercial re-use. See rights and permissions. Published by BMJ.

For numbered affiliations see end of article.

Correspondence to Dr Charlotte Glinge; cglinge@ gmail.com

\section{ABSTRACT}

Objective Morbidity and mortality due to heart failure (HF) as a complication of myocardial infarction (MI) is high, and remains among the leading causes of death and hospitalisation. This study investigated the association between family history of MI with or without HF, and the risk of developing HF after first MI.

Methods Through nationwide registries, we identified all individuals aged 18-50 years hospitalised with first MI from 1997 to 2016 in Denmark. We identified 13810 patients with $\mathrm{MI}$, and the cohort was followed until HF diagnosis, second $\mathrm{Ml}$, 3 years after index $\mathrm{Ml}$, emigration, death or the end of 2016, whichever occurred first. HRs were estimated by Cox hazard regression models adjusted for sex, age, calendar year and comorbidities (reference: patients with no family history of Ml).

Results After adjustment, we observed an increased risk of $\mathrm{Ml}$-induced $\mathrm{HF}$ for those having a sibling with $\mathrm{Ml}$ with $\mathrm{HF}$ (HR 2.05, 95\% Cl 1.02 to 4.12). Those having a sibling with MI without HF also had a significant, but lower increased risk of HF (HR $1.39,95 \% \mathrm{Cl} 1.05$ to 1.84). Parental history of MI with or without HF was not associated with HF.

Conclusion In this nationwide cohort, sibling history of MI with or without HF was associated with increased risk of $\mathrm{HF}$ after first Ml, while a parental family history was not, suggesting that shared environmental factors may predominate in the determination of risk for developing HF.

\section{INTRODUCTION}

Despite significant improvements in the management of acute myocardial infarction (MI) and heart failure (HF), morbidity and mortality due to $\mathrm{HF}$ as a complication of MI remains high, and is one of the leading causes of hospitalisation and death. ${ }^{1-3}$ Therefore, the identification of individuals who are at a high risk of HF after MI represents a key public health necessity. Progression to HF post-MI is likely multifactorial, and includes environmental risk factors, comorbidities and genetic factors. ${ }^{4-7}$ For example, while a similarly sized MI and a comparable exposure to high blood pressure may lead to an effective compensatory response in some patients, a particular constellation of risk factors may result in HF in others. Genetic susceptibility

\section{Key questions}

What is already known about this subject?

- Morbidity and mortality due to heart failure (HF) as a complication of myocardial infarction (MI) is high, and remains among the leading causes of death and hospitalisation.

- It is unknown if a family history of HF after MI is a risk factor for HF post-MI.

What does this study add?

- We demonstrate that having a sibling with MI with or without HF was an independent risk factor for developing $\mathrm{HF}$ after Ml, while a parental family history of MI with or without HF was not.

How might this impact on clinical practice?

- Family history of MI with or without HF, regardless of the nature of the various genetic and environmental factors involved, serves to identify high-risk individuals and families for intervention to prevent HF post-Ml.

factors, environmental exposures and lifestyle factors that contribute to HF susceptibility post-MI are shared within families. Previous studies have suggested a familial aggregation of $\mathrm{HF}^{8-10}$ While this previous finding suggests that inherited susceptibility may contribute to the development of HF post-MI, with the exception of one study, ${ }^{8}$ previous studies did not distinguish between different causes of HF with consequent aetiological heterogeneity. We therefore aimed to determine the association between family history of MI with or without $\mathrm{HF}$ and the risk of developing $\mathrm{HF}$ after a first-time MI in the young (18-50 years of age). This is to our knowledge, the first nationwide study to examine whether a family history of MI with or without HF is associated with $\mathrm{HF}$ among young patients with MI.

\section{METHODS}

\section{Data sources}

A unique and permanent personal identification number is assigned to all residents in Denmark. This number is used in all 
Danish health and administrative registries, and enables individual-level linkage between all nationwide registries. In this study, we combined data from four large Danish registries: 1) The Danish Civil Registration System which contains daily changes in the vital status of all residents, where no historical data are deleted ${ }^{11}$; 2) The Danish National Patient Registry which holds information on all admissions to hospitals since 1978, and outpatient visits since 1995, coded according to the International Classification of Diseases (ICD)-8 and ICD-10 $0^{12}$; 3 ) The Danish Fertility Registry which contains data on almost all parents of children born in Denmark since 1954 and 4) The Danish Registry of Medical Product Statistics, where all prescriptions dispensed from Danish pharmacies have been registered since 1995. Each drug is coded according to the international Anatomical Therapeutic Chemical (ATC) classification system, and the registry includes data on type of drug, strength, quantity dispensed and date of dispensing, ${ }^{13}$ which has been shown to be accurate. ${ }^{14}$

\section{Study population}

The study population comprised all Danish patients aged 18-50 years hospitalised with a first-time MI (ICD-10 code I21 or I22: ICD-8 code 410) between 1997 and 2016. Patients with a prior HF diagnosis (ICD-10 code I42, I50, I110, I130, I132 or J819: ICD-8 code 425, 428, 4270, 4271) were excluded. Using the Danish Fertility Database, we identified all first-degree relatives and collected information on family relationship (mother, father and siblings). Only patients with at least one identifiable first-degree relative (father, mother or sibling) were included.

\section{Family history}

For each individual that was identified with a first MI (index MI) (age 18-50 years), we identified first-degree relatives (aged 18-80 years) with MI with or without HF occurring at a date prior to the MI index date (figure 1). We defined MI with HF as new onset of HF within 3 years from index MI, and MI without HF if the patient did not

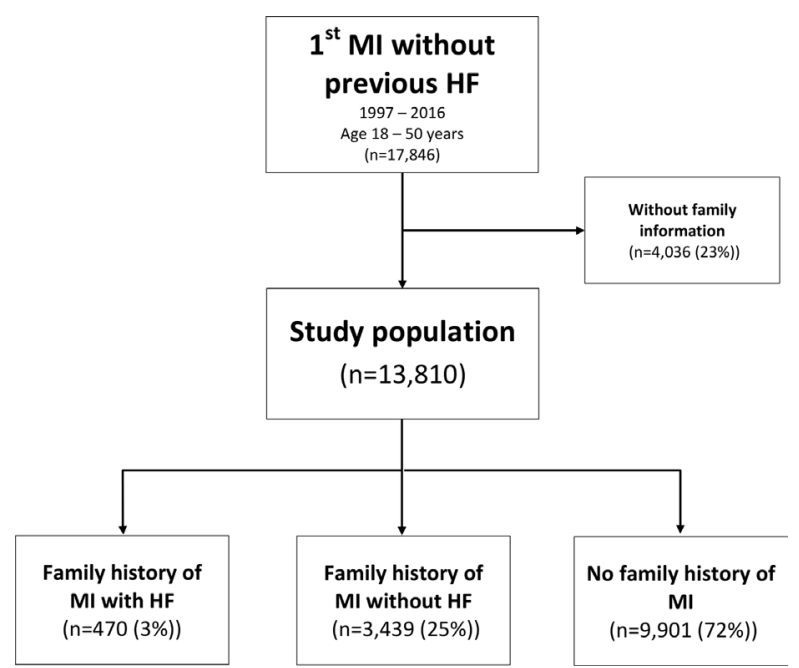

Figure 1 Study population selection. HF, heart failure; MI, myocardial infarction. develop HF in this time period. If no relative was registered as having MI with HF or MI without HF, the person was considered not to have a family history of MI. We subdivided the study population into three groups according to the family history: 1) family history of MI with HF, 2) family history of MI without HF and 3) no family history of MI. Persons with more than one first-degree relative, were still classified as having a family history of MI with HF if they had at least one family member with MI and HF. Family history was further stratified by the family relationship (father, mother or sibling). We additionally analysed whether the risk was similar for patients with a young family member ( $\leq 50$ years at MI) or an old family member with MI with or without HF (51-80 years).

\section{Comorbidities}

Patients were considered to have certain comorbidity if they had at least one hospitalisation registered for that comorbidity before admission for the index MI. Because patients with hypertension (HT) and diabetes (DM) were not necessarily admitted to hospital with these specific diagnoses, these comorbidities were defined by either hospital diagnosis or drug prescription. HT was defined by treatment with $\geq 2$ of the following classes of antihypertensive drugs: $\alpha$-adrenergic blockers, diuretics, vasodilators, $\beta$-blockers, calcium channel blockers and renin-angiotensin system inhibitors. History of DM was additionally identified by at least one prescription for glucose-lowering drugs (ATC code A10). Details on ICD and ATC codes used to classify patients with comorbidity are listed in the online supplementary tables 1 and 2.

\section{Study outcome}

The study population was followed from the date of index MI until the diagnosis of HF, hospitalisation with second MI, date of emigration or death during a maximum of 3 years from the index MI or till study end at 31 December 2016, whichever occurred first. In a sensitivity analysis, we combined the diagnosis of HF and death, as a proxy for a fatal MI.

\section{Statistical analyses}

Baseline characteristics are presented by use of proportions for categorical variables and means $\pm \mathrm{SD}$ for continuous variables. Test for differences between the strata was done by Kruskal-Wallis test for continuous variables and by $\chi^{2}$ test for discrete variables. We produced cumulative incidence curves for HF stratified by family history (figures 2-3) as a competing risk using the AalenJohansen method. Grey tests were used to analyse unadjusted differences. Cox proportional hazard regression models were used to obtain HRs for HF and HF combined with fatal MI, patients with no family history were used as the reference group. The adjusted Cox regression models included sex, age groups (18-35 and $36-50$ years of age), calendar year (1997-2001, 2002-2006, 20072011 and 2012-2016), HT and DM. The proportional hazard assumptions were verified through examination 
2 A

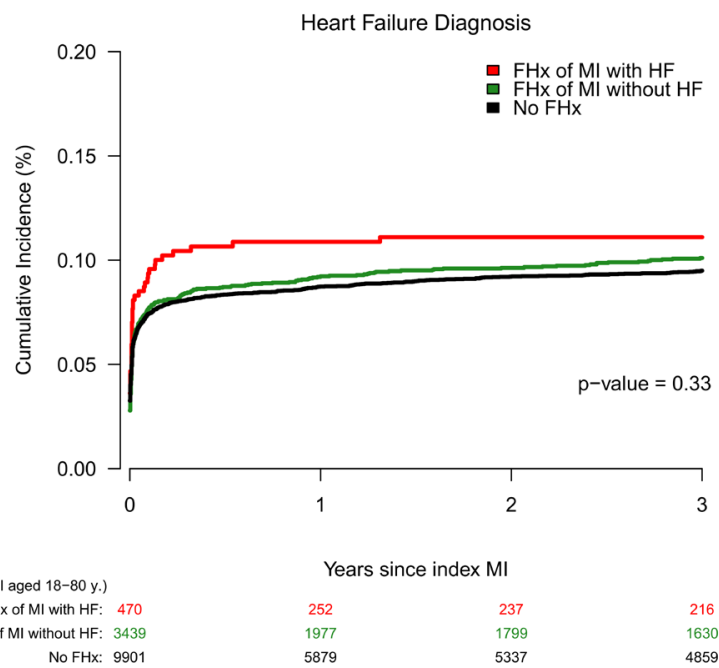

$2 \mathrm{~B}$

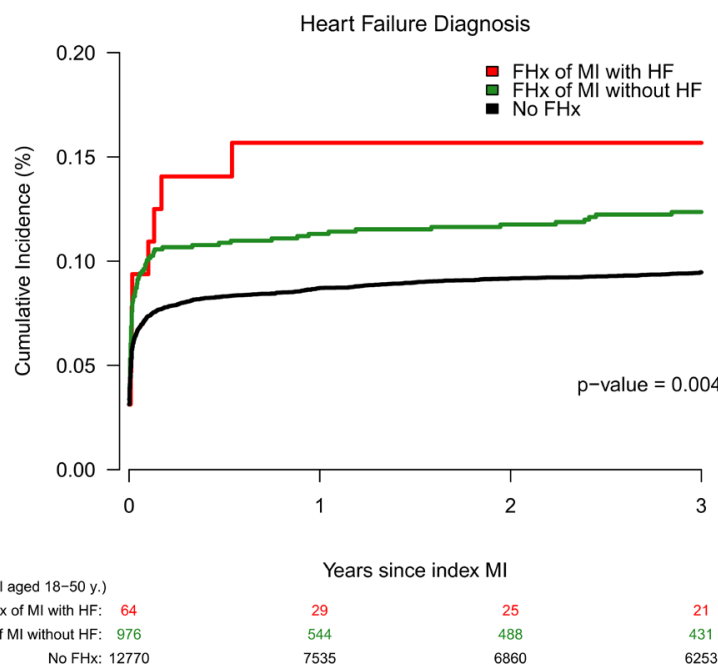

$2 \mathrm{C}$

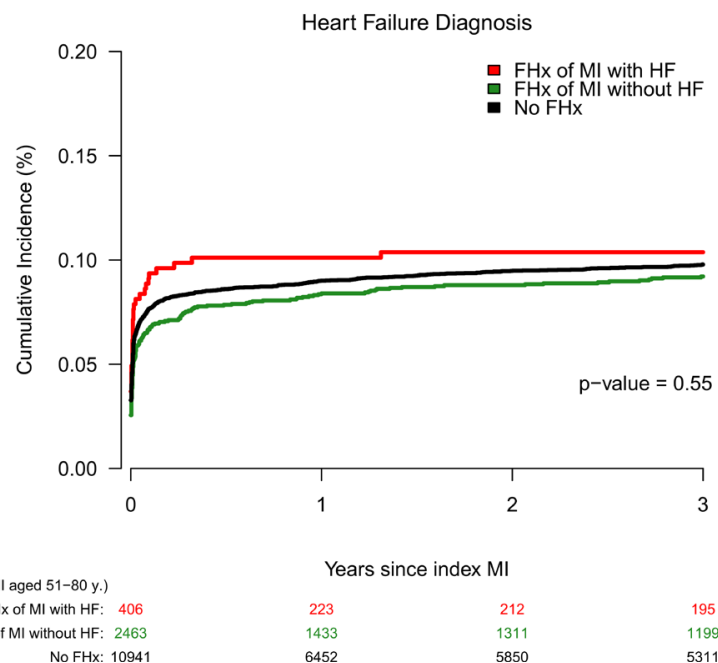

Figure 2 (A-C) Cumulative incidence for heart failure (HF), with death and second myocardial infarction (MI) as competing risk stratified by family history (FHx). FHx was defined as $\mathrm{Ml}$ aged 18-80 years (A), as $\mathrm{Ml}$ aged 18-50 years (B) and as $\mathrm{Ml}$ aged 51-80 years $(\mathrm{C})$.

of Schoenfeld residuals plots. For all analyses, a p value of $<0.05$ was considered statistically significant. All analyses were performed using SAS software, V.9.4 (SAS Institute, Cary, North Carolina, USA), and R V.3.4.

\section{RESULTS}

\section{Family history of MI with or without HF}

We identified 13810 patients aged 18-50 years with firsttime MI hospitalisation between 1997 and 2016, without previous HF hospitalisation, and with $\geq 1$ identifiable firstdegree relative. Figure 1 shows the flow chart of the study population. Of these identified index cases, at inclusion, $470(3 \%)$ had a family history of MI with HF, 3439 (25\%) had a family history of MI without HF and the remaining $9901(72 \%)$ had no family history of MI.

\section{Clinical characteristics}

Table 1 shows the baseline characteristics of the index cases at study inclusion stratified by family history. Overall, there was a male predominance in all groups and significant more males had a family history of MI. Patients with a family history of MI with or without HF were older (mean age 45 and 44 years vs 42 years) and had more HT (24\% and $21 \%$, respectively), DM $(12 \%$ and $10 \%$, respectively) and a higher use of statins (20\% and $17 \%$, respectively) compared with patients without any family history of MI (19\%, $8 \%$ and $12 \%)$.

\section{Incidence of HF after first MI}

A total of 1324 patients $(10 \%)$ were diagnosed with HF during the follow-up period (mean follow-up 1.7 years). The mean time of HF diagnosis after index MI was 74 days. The cumulative incidence of $\mathrm{HF}$ after first MI was 11.1\% (95\% CI 8.3 to 14.0$), 10.1 \%(95 \%$ CI 9.1 to 11.1$)$ and $9.5 \%$ (95\% 


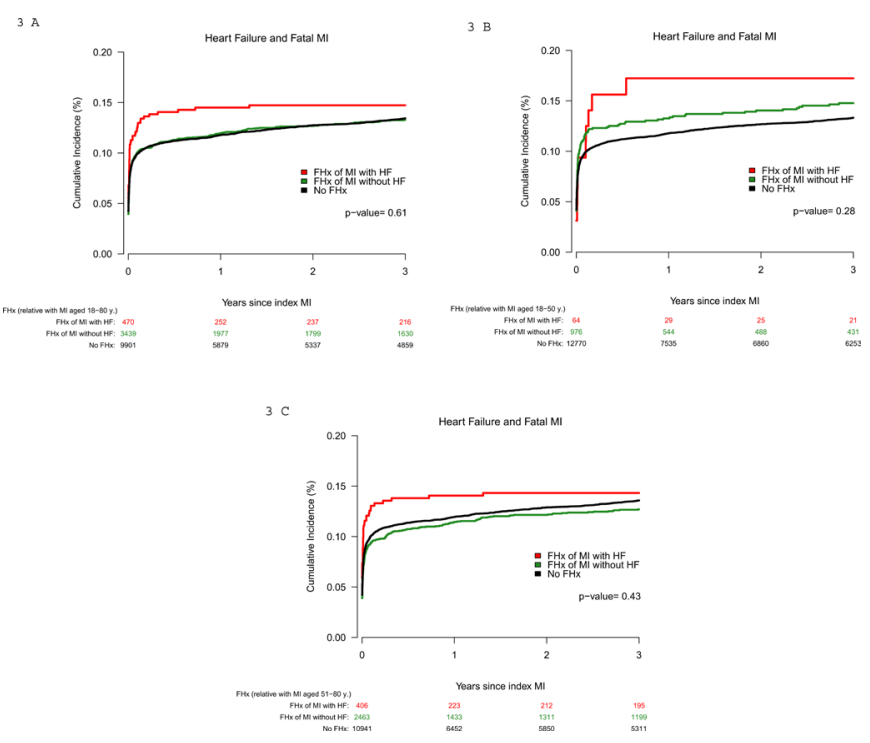

Figure $3(\mathrm{~A}-\mathrm{C})$ Cumulative incidence for heart failure (HF) and fatal myocardial infarction (MI), with second $\mathrm{MI}$ as competing risk, stratified by family history $(\mathrm{FHx})$. $\mathrm{FHx}$ was defined as $\mathrm{Ml}$ aged $18-80$ years $(\mathrm{A})$, as $\mathrm{Ml}$ aged $18-50$ years (B) and as $\mathrm{Ml}$ aged $51-80$ years (C).

CI 8.9 to 10.1 ) in patients with a family history of MI with HF, MI without HF and no family history of MI, respectively, as illustrated in figure 2A. Family history was further stratified by the age at MI of the first-degree relative (younger vs above 50 years of age) (figure 2B-C). In addition, the cumulative incidences were repeated with a combined outcome of HF diagnosis and fatal MI, but yielded similar results as the main analysis with $\mathrm{HF}$ as outcome, except that family history of a young first-degree relative ( $\leq 50$ years) was no longer significant (figure 3A-C).

\section{Risk of HF stratified according to family history of MI with or without HF}

Figure 4 presents the crude (figure $4 \mathrm{~A}$ ) and the adjusted $\mathrm{HR}$ (figure $4 \mathrm{~B}$ ) for family history of $\mathrm{MI}$ with $\mathrm{HF}$ and family history of MI without HF compared with no family history, stratified by family relationship. Having a sibling with MI complicated by HF was associated with a twofold increased risk of $\mathrm{HF}$ after first MI compared with cases without a sibling with MI (HR 2.05, 95\% CI 1.02 to 4.12). In addition, having a sibling with $\mathrm{MI}$ without $\mathrm{HF}$ was associated with increased HF risk (HR 1.39, 95\% CI 1.05 to 1.84). However, having a mother or father with MI with or without HF was not significantly associated with HF risk after first MI. The HRs were repeated with a combined outcome of HF diagnosis and fatal MI (figure 5). The HF risk associated with family history of MI with or without HF was similar regardless of whether the relative with MI was young ( $\leq 50$ years) (online supplementary figure 1A-B) or old (51-80 years) (online supplementary figure 2A-B).

\section{DISCUSSION}

In this nationwide study, we investigated the relationship between family history of MI with or without $\mathrm{HF}$ and the risk of developing $\mathrm{HF}$ after a first $\mathrm{MI}$ in individuals 18-50 years of age. We demonstrate that having a sibling with MI with or without HF was an independent risk factor for

Table 1 Baseline characteristics of the study population $(n=13810)$

\begin{tabular}{|c|c|c|c|c|c|}
\hline Variable & Total $(n=13810)$ & FHx of MI with HF $(n=470)$ & FHx of MI without HF $(n=3439)$ & $\begin{array}{l}\text { No FHx } \\
(n=9901)\end{array}$ & $P$ value \\
\hline Sex, male, $n(\%)$ & $10344(74.9)$ & $350(74.5)$ & $2662(77.4)$ & $7332(74.1)$ & $<0.001$ \\
\hline Ml age, mean (SD) & $42.8(5.7)$ & $44.6(4.2)$ & $43.8(4.9)$ & $42.4(6.0)$ & $<0.0001$ \\
\hline \multicolumn{6}{|l|}{ Age group (years), $n(\%)$} \\
\hline $18-35$ & $1649(11.9)$ & $23(4.9)$ & $254(7.4)$ & $1372(13.9)$ & \multirow[t]{2}{*}{$<0.0001$} \\
\hline $36-50$ & $12161(88.1)$ & $447(95.1)$ & $3185(92.6)$ & $8529(86.1)$ & \\
\hline \multicolumn{6}{|l|}{ Calendar year, n (\%) } \\
\hline 1997-2001 & $2316(16.8)$ & $76(16.2)$ & $567(16.5)$ & $1673(16.9)$ & \multirow[t]{4}{*}{0.977} \\
\hline 2002-2006 & $3924(28.4)$ & $135(28.7)$ & $964(28.0)$ & $2825(28.5)$ & \\
\hline $2007-2011$ & $3838(27.8)$ & $131(27.9)$ & $976(28.4)$ & $2731(27.6)$ & \\
\hline 2012-2016 & $3732(27.0)$ & $128(27.2)$ & $932(27.1)$ & $2672(27.0)$ & \\
\hline HT, n (\%) & $2637(19.1)$ & $113(24.0)$ & 733 (21.3) & $1791(18.1)$ & $<0.0001$ \\
\hline Statin use, n (\%) & 1848 (13.4) & $96(20.4)$ & $589(17.1)$ & $1163(11.7)$ & $<0.0001$ \\
\hline $\mathrm{DM}, \mathrm{n}(\%)$ & $1214(8.8)$ & $55(11.7)$ & $329(9.6)$ & $830(8.4)$ & 0.008 \\
\hline Kidney disease, n (\%) & 441 (3.2) & $13(2.8)$ & $101(2.9)$ & 327 (3.3) & 0.499 \\
\hline AF, n (\%) & $239(1.7)$ & $4(0.9)$ & $46(1.3)$ & $189(1.9)$ & 0.029 \\
\hline PVD, n (\%) & $215(1.6)$ & $7(1.5)$ & $65(1.9)$ & $143(1.4)$ & 0.190 \\
\hline Valve disease, $\mathrm{n}(\%)$ & $152(1.1)$ & $7(1.5)$ & $33(1.0)$ & $112(1.1)$ & 0.505 \\
\hline
\end{tabular}

AF, atrial fibrillation; DM, diabetes; FHx, family history; HT, hypertension; MI, myocardial infarction; PVD, peripheral vascular disease. 
A

\begin{tabular}{|c|c|c|c|c|c|}
\hline Family History (FHx) & Individuals (events) & & HR & $95 \% \mathrm{Cl}$ & P-value \\
\hline Sibling & $11,697(1,124)$ & & & & \\
\hline MI with $\mathrm{HF}$ & $32(8)$ & $\longrightarrow$ & 2.73 & $1.36-5.46$ & $<0.01$ \\
\hline Ml without HF & $371(51)$ & 를- & 1.51 & $1.14-2.00$ & $<0.01$ \\
\hline No $F H x$. of MI & $11,294(1,065)$ & = & 1.00 & Reference & \\
\hline Father & $13,071(1,252)$ & & & & \\
\hline MI with $\mathrm{HF}$ & $296(37)$ & - - & 1.37 & $0.99-1.91$ & 0.06 \\
\hline MI without HF & 2,394 (234) & ㅁ. & 1.04 & $0.90-1.20$ & 0.58 \\
\hline No FHx. of MI & 10,381 (981) & a & 1.00 & Reference & \\
\hline Mother & $13,650(1,312)$ & & & & \\
\hline MI with HF & $152(9)$ & $=-$ & 0.62 & $0.32-1.20$ & 0.16 \\
\hline MI without HF & $1,102(104)$ & E & 0.98 & $0.80-1.19$ & 0.81 \\
\hline No FHx. of Ml & $12,396(1,199)$ & ${ }_{15}^{1}$ & 1.00 & Reference & \\
\hline
\end{tabular}

B

\begin{tabular}{|c|c|c|c|c|c|}
\hline Family History $(\mathrm{FHx})$ & Individuals (events) & & $\mathrm{HR}^{*}$ & $95 \% \mathrm{Cl}$ & P-value \\
\hline Sibling & $11,697(1,124)$ & & & & \\
\hline MI with $\mathrm{HF}$ & $32(8)$ & $\longrightarrow$ & 2.05 & $1.02-4.12$ & 0.04 \\
\hline MI without HF & $371(51)$ & 훌 & 1.39 & $1.05-1.84$ & 0.02 \\
\hline No FHx. of MI & $11,294(1,065)$ & - & 1.00 & Reference & \\
\hline Father & $13,071(1,252)$ & & & & \\
\hline MI with $\mathrm{HF}$ & $296(37)$ & $=$ & 1.37 & $0.99-1.90$ & 0.06 \\
\hline MI without $\mathrm{HF}$ & $2,394(234)$ & - & 1.02 & $0.88-1.18$ & 0.80 \\
\hline No FHx. of MI & $10,381(981)$ & a & 1.00 & Reference & \\
\hline Mother & $13,650(1,312)$ & & & & \\
\hline MI with $\mathrm{HF}$ & $152(9)$ & -2 & 0.60 & $0.31-1.15$ & 0.13 \\
\hline MI without $\mathrm{HF}$ & $1,102(104)$ & $\mathbf{E}$ & 0.96 & $0.78-1.17$ & 0.68 \\
\hline No FHx. of MI & $12,396(1,199)$ & 1 & 1.00 & Reference & \\
\hline
\end{tabular}

Figure 4 (A, B) HRs for heart failure (HF) according to family history. Crude (A) and adjusted (B) for sex, age group, calendar year group, history of hypertension and diabetes. MI, myocardial infarction. *Adjusted for covariates.

developing HF after MI, while a parental family history of MI with or without HF was not.

\section{Family aggregation}

We found that while sibling history was associated with HF risk, parental history was not. Differences in association between siblings and parent-offspring could indicate that environmental factors shared by siblings are more important in determining susceptibility to HF compared with inherited (genetic) contributions. ${ }^{15} 16$ Aggregation among siblings suggests that early life familial environmental exposures may increase HF risk, because siblings share the family environment at similarly young ages, as opposed to children and parents whose ages generally differ by two to four decades. In line with the observations in this study, previous studies have shown that siblingsibling correlations for most coronary artery disease (CAD) risk factors are larger than parent-offspring correlations. $^{15}$

Our findings contrast to those obtained in two previous studies that investigated risk for all-cause HF and demonstrated that a parental history of HF was associated with increased risk of $\mathrm{HF}$ in the offspring. ${ }^{89}$ Specifically, data from the Framingham Offspring Study ( $\mathrm{n}=2214)$ showed that the occurrence of HF in at least one parent (occurring before 75 years of age) was a significant risk factor for HF in the offspring $(n=90) .{ }^{8}$ In subanalyses, parental
A

Family History (FHx)
Sibling
MI with HF
MI without HF
No FHx. of MI
Father
MI with HF
MI without HF
No FHx. of MI
Mother
MI with HF
MI without HF
No FHx. of MI
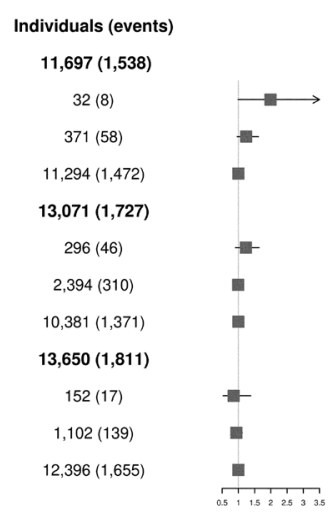

HR $\quad 95 \% \mathrm{Cl}$

B Family History (FHx)
Sibling
MI with HF
Ml without HF
No FHx. of MI
Father
MI with HF
MI without HF
No FHx. of MI
Mother
MI with HF
MI without HF
No FHx. of MI
Individuals (events)
$11,697(1,538)$
32 (8)
371 (58)
$11,294(1,472)$
$13,071(1,727)$
296 (46)
$2,394(310)$
$10,381(1,371)$
$13,650(1,811)$
$152(17)$
$1,102(139)$
$12,396(1,655)$

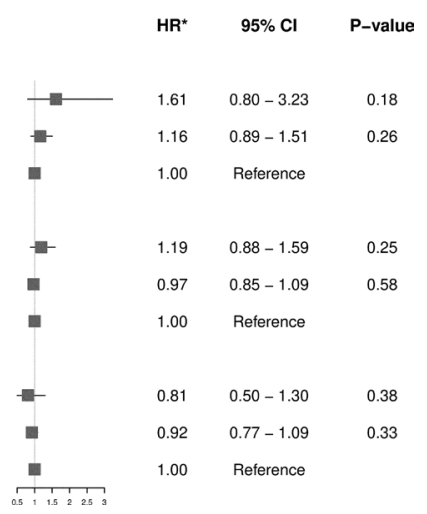

Figure 5 (A, B) HRs for heart failure (HF) and fatal myocardial infarction (MI) according to family history. Crude (A) and adjusted (B) for sex, age group, calendar year group, history of hypertension and diabetes. *Adjusted for covariates.

ischaemic $\mathrm{HF}$ was also associated with risk of ischaemic $\mathrm{HF}$ in the offspring ( $\mathrm{n}=11)$ (HR 2.46, 95\% CI 1.10 to 5.49). The other is a recent Swedish adoption study of 21643 adoptees, of whom 194 developed HF, which found that the risk of HF in adoptees who had at least one biological parent with $\mathrm{HF}$ was $45 \%$ higher than in a control group with no biological parent suffering from $\mathrm{HF}^{9}{ }^{9}$ In addition, this study found no increased risk in individuals with adoptive parents with HF. Crucially, these earlier studies investigated a smaller group of index cases compared with the current analysis. Furthermore, unlike our study that focused specifically on HF post-MI in both the index cases and the first-degree relatives, these studies (with the exception of the subanalysis conducted in a limited number of patients in the first study) did not distinguish between causes of HF in neither the parents nor the offspring. Although CAD is one of the most common causes of $\mathrm{HF}^{17}$ the well-known monogenic forms of $\mathrm{HF}$ could partly explain the familial aggregation in these previous studies, and could underlie, at least in part, the findings of parental history as a risk factor for HF in the offspring. Additionally, our study has the advantage that it was conducted in a nationwide fashion collecting family history and relationship data nationwide. 
The genetic architecture underlying susceptibility for ischaemic HF is unknown but is believed to comprise multiple genetic variants that in combination with environmental factors determine an individual's susceptibility to HF. While large-scale genome-wide association studies (GWAS) have been successful in identifying common genetic variants affecting CAD/MI risk, ${ }^{18}$ GWAS studies for $\mathrm{HF}$ are sparse in the literature and compared with other complex cardiovascular traits, few common susceptibility genetic variants have been uncovered for HF thus far. ${ }^{19}$ The largest GWAS study on incident HF, conducted on $2526 \mathrm{HF}$ events, identified only two single nucleotide polymorphisms. ${ }^{20}$ The paucity of identified genetic susceptibility factors for HF can be due to several factors including limited statistical power due to the relatively small number of patients with HF that have been studied by GWAS so far as well as the heterogeneity in the causes of HF in the patients studied. However, this could also point to the fact that genetic factors may not play a major role in the determination of risk for HF and that other factors, such as environmental factors, predominate in the determination of risk. Our finding that a sibling history of HF post-MI is a risk factor for HF whereas a parental history is not, supports the latter scenario. On the other hand, our data do not exclude the possibility that inherited predisposition for HF exists but only leads to HF in the presence of certain environmental factors. As such, genetic variants could influence risk for HF through comorbidities that predispose to $\mathrm{HF}^{21-24}$ We found that index cases with a family history of MI with or without HF were older, had higher prevalence of HT and DM, both well-known contributing risk factors for $\mathrm{MI}$ and $\mathrm{HF}^{25}$ compared with patients with no family history of MI. One could hypothesise that as genetic factors predisposing to comorbidities contributing to HF become known, approaches such as polygenic risk score analysis will enable the presymptomatic identification of susceptible individuals and will allow for modification of environmental factors and lifestyle adjustments to prevent HF. Nonetheless, family history of MI with or without HF, regardless of the nature of the various genetic and environmental factors involved, serves to identify high-risk individuals and families for intervention to prevent HF post-MI.

The recognition of these complexities mentioned above should impact the design of future studies seeking to identify genetic and environmental risk factors of MI-induced HF. Studies should therefore focus on a welldefined phenotype (eg, HF following a first MI) instead of all-cause HF. Large GWAS of CAD and subsequent events are ongoing, and are expected to yield more insights into the contribution of heritable risk factors to HF post-MI. ${ }^{26}$

\section{Strengths and limitations}

The major strengths of our study include the large and unselected nationwide cohort of index cases 18-50 years with a first MI, the extensive information on family history, comorbidity and pharmacotherapy and the combination of detailed data, keeping selection bias at a minimum.
The main limitations of the study were the time frame in which family relations could be determined (the identity of parents has been available in Denmark since 1954) and the time frame in which the diagnoses were registered (1978-2016). In addition, as the parents were born a few decades earlier than the offspring and siblings there is the possibility that due to diagnostic limitations, HF may have gone unrecognised and undiagnosed. Furthermore, it is well known that MI occurs up to 10 years later in women compared with men, ${ }^{2}$ and that HF is more likely to complicate MI in older persons. This could mean that the mother of the index case might have been too young and have not yet developed MI. This could possibly lead to an underestimation of the number of mothers with MI (with or without $\mathrm{HF}$ ), which may explain the lack of association between maternal history of MI (with or without HF) and the risk of $\mathrm{HF}$ in this study. In addition, the method of identifying HF with ICD codes does not identify patients with unrecognised HF. Because of this, HF may be underdiagnosed in our registries. On the other hand, the risk of including false-positive patients is limited, as previous validation of $\mathrm{HF}$ diagnosis by Kümler et al showed a positive predictive value of $81 \% .{ }^{27}$ Of note, the study reported a sensitivity of HF diagnosis of $29 \%$, which indicates a large underestimation of the true number of HF cases. Additionally, with only a total of 470 index cases with a family history of MI with HF and the small numbers of affected offspring and siblings, the study might not have sufficient statistical power to evaluate the effect on the risk of HF post-MI. Other limitations are inherited to the observational design of the study and the lack of access to clinical data on individual patient characteristics, most importantly the type of MI, including the localisation of MI, left ventricular ejection fraction and information of other important cardiovascular risk factors. These clinical variables may be confounders, because these risk factors tend to cluster in families, and therefore might have influence on the HF risk after first-time MI in families. ${ }^{28}{ }^{29}$ On the other hand, it is possible that an ascertainment bias may have occurred with individuals with a positive sibling history of MI with or without HF as these may have a greater likelihood of diagnoses (HT, DM and use of statins) than participants with no family history, due to increased awareness of the condition by participants. Finally, our study is observational and retrospective, thus we report associations and not necessarily causal connections.

\section{CONCLUSION}

In this nationwide cohort study of young patients (18-50 years of age) with a first MI, we found that having a sibling with MI with or without HF was associated with increased risk of HF post-MI while a parental family history of MI with or without HF was not, suggesting that shared environmental factors predominate in the determination of risk for developing HF. Future studies are necessary to validate the kinds of conclusions that can be drawn 
from this study. This study provides important knowledge for the planning of future studies to untangle risk factor relationships and enhancing our understanding of the underlying mechanisms of HF after first MI. Better understanding of the underlying mechanisms of $\mathrm{HF}$ post-MI will improve our current risk stratification, diagnosis, intervention and prevention.

\section{Author affiliations}

${ }^{1}$ Department of Cardiology, The Heart Centre, Copenhagen University Hospital, Rigshospitalet, Copenhagen, Denmark

${ }^{2}$ Department of Clinical and Experimental Cardiology, Amsterdam UMC, University of Amsterdam, Heart Center, Amsterdam Cardiovascular Sciences, Amsterdam, The Netherlands

${ }^{3}$ Department of Cardiology, Gentofte Hospital, University of Copenhagen, Hellerup, Denmark

${ }^{4}$ Department of Health, Science and Technology, Aalborg University, Aalborg, Denmark

${ }^{5}$ Department of Cardiology, University of Lund, Lund, Sweden

${ }^{6}$ The National Institute of Public Health, University of Southern Denmark, Copenhagen, Denmark

${ }^{7}$ The Danish Heart Foundation, Copenhagen, Denmark

${ }^{8}$ Department of Forensic Medicine, Faculty of Medical Sciences, University of Copenhagen, Copenhagen, Denmark

Acknowledgements The authors would like to thank Novo Nordisk Foundation and the University Hospital of Copenhagen, Rigshospitalet for financial support.

Contributors CG performed the analysis and drafted the manuscript. RS verified the analytical methods. LO, RJ, CB, CT-P, GG and JT-H were involved in planning and supervised the work. SR, LK and TE aided in interpreting the results and worked on the manuscript. All authors discussed the results, provided critical feedback and commented on the manuscript.

Funding This work was supported by the Novo Nordisk Foundation, and CG was funded by the University Hospital of Copenhagen, Rigshospitalet, Copenhagen, Denmark.

Competing interests None declared.

Patient consent for publication Not required.

Ethics approval The study was approved by the Danish Data Protection Agency (ref. number: 2007-58-0015/GEH-2014-014 and I-suite number: 02732). In Denmark, ethical approval is not required for retrospective register-based studies.

Provenance and peer review Not commissioned; externally peer reviewed.

Data availability statement All data relevant to the study are included in the article or uploaded as supplementary information.

Open access This is an open access article distributed in accordance with the Creative Commons Attribution Non Commercial (CC BY-NC 4.0) license, which permits others to distribute, remix, adapt, build upon this work non-commercially, and license their derivative works on different terms, provided the original work is properly cited, appropriate credit is given, any changes made indicated, and the use is non-commercial. See: http://creativecommons.org/licenses/by-nc/4.0/.

ORCID ID

Charlotte Glinge http://orcid.org/0000-0003-0406-256X

\section{REFERENCES}

1 Gerber Y, Weston SA, Enriquez-Sarano M, et al. Atherosclerotic burden and heart failure after myocardial infarction. JAMA Cardiol 2016;1:156-62.

2 Benjamin EJ, Blaha MJ, Chiuve SE, et al. Heart disease and stroke Statistics-2017 update. Circulation 2017;135:e146-603.

3 Vasan RS, Xanthakis V, Lyass A, et al. Epidemiology of Left Ventricular Systolic Dysfunction and Heart Failure in the Framingham Study: An Echocardiographic Study Over 3 Decades. JACC Cardiovasc Imaging 2018;11:1-11.

4 Lewis EF, Velazquez EJ, Solomon SD, et al. Predictors of the first heart failure hospitalization in patients who are stable survivors of myocardial infarction complicated by pulmonary congestion and/or left ventricular dysfunction: a VALIANT study. Eur Heart $J$ 2008;29:748-56.

5 Gho JMIH, Postema PG, Conijn M, et al. Heart failure following STEMI: a contemporary cohort study of incidence and prognostic factors. Open Heart2017;4:e000551.

6 Verweij N, Eppinga RN, Hagemeijer Y, et al. Identification of 15 novel risk loci for coronary artery disease and genetic risk of recurrent events, atrial fibrillation and heart failure. Sci Rep 2017;7:2761.

7 Andersson $\mathrm{C}$, Lyass A, Lin $\mathrm{H}$, et al. Association of genetic variation in coronary artery Disease-Related loci with the risk of heart failure with preserved versus reduced ejection fraction. Circulation 2018;137:1290-2.

8 Lee DS, Pencina MJ, Benjamin EJ, et al. Association of parental heart failure with risk of heart failure in offspring. $N$ Engl J Med 2006;355:138-47.

9 Lindgren MP, PirouziFard M, Smith JG, et al. A Swedish nationwide adoption study of the heritability of heart failure. JAMA Cardiol 2018;3:703-10.

10 Lindgren MP, Smith JG, Li X, et al. Sibling risk of hospitalization for heart failure - a nationwide study. Int J Cardiol 2016;223:379-84.

11 Schmidt M, Pedersen L, Sørensen HT. The Danish civil registration system as a tool in epidemiology. Eur J Epidemiol 2014;29:541-9.

12 Schmidt M, Schmidt SAJ, Sandegaard JL, et al. The Danish national patient registry: a review of content, data quality, and research potential. Clin Epidemiol 2015;7:449-90.

13 Pottegård A, Schmidt SAJ, Wallach-Kildemoes H, et al. Data resource profile: the Danish national prescription registry. Int $J$ Epidemiol 2017;46:798-798f.

14 Rasmussen L, Valentin J, Gesser KM, et al. Validity of the prescriber information in the Danish national prescription registry. Basic Clin Pharmacol Toxicol 2016;119:376-80.

15 Knuiman MW, Divitini ML, Welborn TA, et al. Familial correlations, cohabitation effects, and heritability for cardiovascular risk factors. Ann Epidemiol 1996;6:188-94.

16 Murabito JM, Pencina MJ, Nam B-H. Sibling cardiovascular disease as a risk factor for cardiovascular disease in middle-aged adults. JAMA 2005;294:3117-23.

17 Metra M, Teerlink JR. Heart failure. Lancet 2017;390:1981-95.

18 Erdmann J, Kessler T, Munoz Venegas L, et al. A decade of genomewide association studies for coronary artery disease: the challenges ahead. Cardiovasc Res 2018;114:1241-57.

19 Buniello A, MacArthur JAL, Cerezo M, et al. The NHGRI-EBI GWAS Catalog of published genome-wide association studies, targeted arrays and summary statistics 2019. Nucleic Acids Res 2019;47:D1005-12.

20 Smith NL, Felix JF, Morrison AC, et al. Association of genome-wide variation with the risk of incident heart failure in adults of European and African ancestry: a prospective meta-analysis from the cohorts for heart and aging research in genomic epidemiology (CHARGE) consortium. Circ Cardiovasc Genet 2010;3:256-66.

21 Warren HR, Evangelou E, Cabrera CP, et al. Genome-wide association analysis identifies novel blood pressure loci and offers biological insights into cardiovascular risk. Nat Genet 2017;49:403-15

22 Mahajan A, Taliun D, Thurner M, et al. Fine-mapping type 2 diabetes loci to single-variant resolution using high-density imputation and islet-specific epigenome maps. Nat Genet 2018;50:1505-13.

23 Nielsen JB, Thorolfsdottir RB, Fritsche LG, et al. Biobank-driven genomic discovery yields new insight into atrial fibrillation biology. Nat Genet 2018;50:1234-9.

24 Roselli C, Chaffin MD, Weng L-C, et al. Multi-ethnic genome-wide association study for atrial fibrillation. Nat Genet 2018;50:1225-33.

25 Bozkurt B, Aguilar D, Deswal A, et al. Contributory risk and management of comorbidities of hypertension, obesity, diabetes mellitus, hyperlipidemia, and metabolic syndrome in chronic heart failure: a scientific statement from the American heart association. Circulation 2016;134:e535-78.

26 Patel RS, Asselbergs FW. The GENIUS-CHD Consortium. Eur Heart J 2015;36:2674-6.

27 Kümler T, Gislason GH, Kirk V, et al. Accuracy of a heart failure diagnosis in administrative registers. Eur J Heart Fail 2008;10:658-60.

28 Rissanen AM, Nikkilä EA. Aggregation of coronary risk factors in families of men with fatal and non-fatal coronary heart disease. $\mathrm{Br}$ Heart J 1979;42:373-80.

29 Chen W, Srinivasan SR, Bao W, et al. The magnitude of familial associations of cardiovascular risk factor variables between parents and offspring are influenced by age: the Bogalusa heart study. Ann Epidemiol 2001;11:522-8. 